\title{
The Development of Nereis pelagica Linnæus.
}

\author{
By
}

\author{
Douglas P. Wilson, M.Sc.,
}

Assistant Naturalist at the Plymouth Laboratory.

With 12 Figures in the Text.

\section{CONTENTS.}

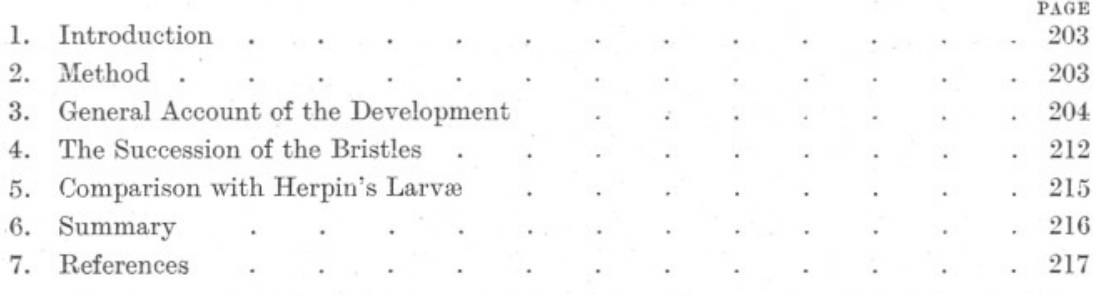

\section{Introduction.}

Although a great deal of work has been done on the development of various species of the Nereids, and the general outlines of the development of a Nereid worm are well known, there still remain several species about which we have comparatively little information. Such a species is Nereis pelagica Linn. which appears to have been reared previously by only one worker, Herpin (1926), who, however, did not get it to grow past a comparatively early stage, and whose experience differed considerably from mine. These differences will be pointed out at the end of the paper.

The work of which this is a record was done five years ago. Publication has been delayed by various causes, one being the hope that it might be possible to repeat the rearing and so add various details to the description. An opportunity for repetition not having presented itself the account is given as it stands.

\section{Method.}

The larvæ were reared in a plunger-jar filled with outside sea-water which had been filtered through fine bolting silk. By the time the young worms were ready to feed the jar contained a good growth of diatoms. Larvæ were examined in cavity slides without pressure and while free to 
move about. A drop of saliva-as mentioned by Herpin-added to the sea-water in the cavity slide proved very effective in slowing down the movements of the active creatures without in any way killing or distorting them. In such a solution they survived for hours and after having been washed free from it lived for days in finger bowls. Such larvæ, however, were never returned to the plunger-jar. This saliva method has not proved successful in the case of any other Polychæte larvæ which I have tried. The drawings were all made from living specimens-frequently while confined in saliva - with the aid of a squared net micrometer, drawing in the first place on to squared paper. These drawings were then checked, as far as possible, from specimens fixed in Bouin and mounted in Canada Balsam. Bristles were examined in Farrant's Medium.

\section{General Account of the Development.}

On the afternoon of 4th February, 1927, Mr. William Searle collected on the shore at Rum Bay three heteronereids of Nereis pelagica, two males and one female. These were placed in a dish in the Laboratory. During the late afternoon one of the males showed considerable activity in swimming round and round the dish and was still doing so at 10 p.m. The other two worms rested quietly on the bottom. The following morning both males were swimming rapidly, the female resting, apparently exhausted, on the bottom. She had spawned and the water was milky with countless eggs. Some of these were put into finger bowls containing fresh outside sea-water. In order to make sure that the eggs were fertilized the males were cut open and a little of the sperm which gushed out through the cuts was added to them. Some of the eggs were then transferred to a plunger-jar. The female was also cut open and a few remaining eggs shaken out into clean sea-water and sperm added in the usual way when making an artificial fertilization.

The eggs contained a large number of oily-looking globules which in some cases were observed to run together to form fewer larger globules. Each egg was about $180 \mu$ in diameter and lay in a spherical cavity about $225 \mu$ in diameter enclosed by a very thick (about $150 \mu$ ) gelatinous and very transparent envelope, the inner boundary of which was quite distinct but the outer difficult to see. As Herpin (1926) has pointed out this jelly is not adhesive as is the case with Perinereis cultrifera and $P$. marioni, in which species it sticks the eggs to the side of the bowls and possibly to rocks. In $N$. pelagica it is almost certain that the eggs are pelagic.

The eggs which had been naturally spawned segmented during the morning, but those which were artificially fertilized did not show the first cleavage until five hours afterwards. Late on February 6th a good proportion of the embryos began to rotate, pressing themselves against the 
inner surface of the gelatinous envelope. This they continued to do during the following day. On February 8th, three and a half days after fertilization, the majority hatched out, a number having done so some hours previously. They swam actively and often a thin membrane, apparently the last remains of the egg envelope, clung to them for a time before being finally thrown off. The fate of the gelatinous main mass of the envelope was unfortunately not made out.

A lateral view of a recently hatched larva is shown in Figure 1. In essentials it corresponded exactly to the free swimming type of Nereis larva known in such detail from the work of E. B. Wilson (1892) and others. Three pairs of parapodia were present, each parapodium having noto- and neuropodial chæta-sacs which contained chætæ whose tips

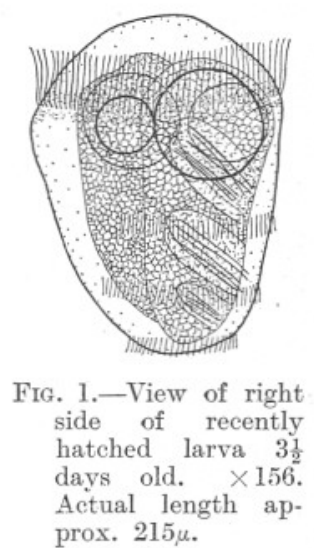

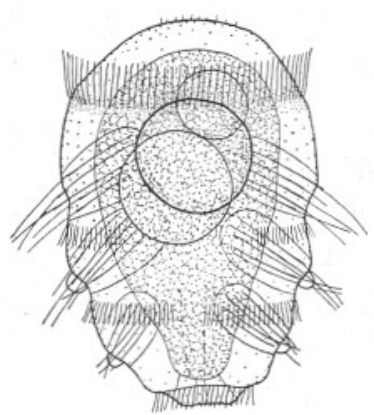

Fig. 2.-Dorsal view oî larva about $4 \frac{1}{2}$ days old. $\times 156$. Actual length approx. $250 \mu$.

already protruded to the exterior. The prototroch was a complete ring of cilia, arranged in a single row. Underlying it was an irregular band of pale pink pigment. Three paratrochs were present, the first two with dorsal gaps, the anterior possessing the largest of these. The third paratroch was almost a complete ring, but a slight dorsal gap may have been present. As will be apparent later it was not a true telotroch. It was suspected that the single row of fine cilia forming each of these paratrochs was itself broken up into short rows of cilia placed end to end but with slight gaps between the ends. Four large and conspicuous oil globules of differing size were situated anteriorly. Four was the usual number of these globules, but the number as well as the size varied. The internal structure was not clear, the region of the gut being granular. A day later (Fig. 2) the chætæ protruded for a considerable distance and in the second and third pairs of parapodia a lobe was growing out between the noto- and neuropodial sacs. A few short cilia, possibly sensory, had appeared at the anterior extremity. The dorsal gap in the second para- 
troch was narrower than previously. The third paratroch had a ventral gap. Another day later and the condition shown in Figure 3 was reached. The chætæ were really long and distinctly articulated. Two pairs of pinkish eye-spots had appeared and anterior to them a row of cilia on each side formed an akrotroch. The dorsal gap in the second paratroch

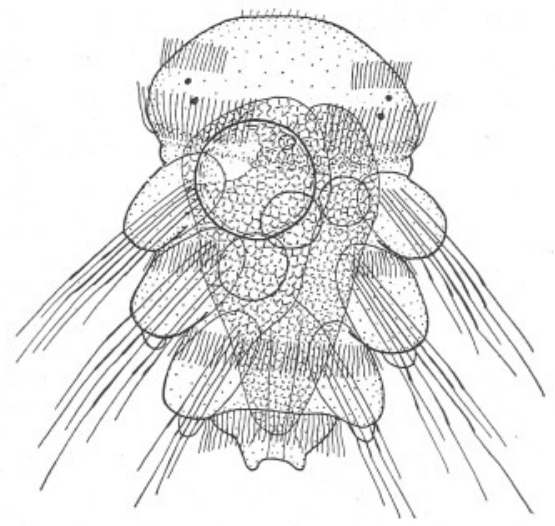

Fig. 3.-Dorsal view of larva about 5 days old. $\times 156$. Actual length approx. $286 \mu$.

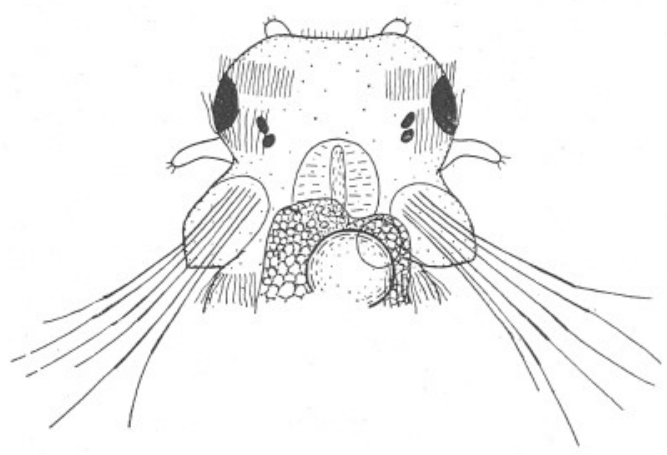

Fia. 4.-Dorsal view of head of larva about 7 days old. $\times 156$.

had closed up, but it now had a ventral one as had also the first and third. The dorsal and ventral portions of the prototroch had each disappeared for a short distance, thereby dividing that organ into lateral, albeit still lengthy, portions. The pink pigment in the prototrochal region still formed a conspicuous speckled band. Anal cirri were appearing and the mouth could be distinguished (about one o'clock of the large oil-globule in Figure 3). The following day the first signs of the tentacles were seen 
at the anterior end of the prostomium and the first pair of tentacular cirri were visible as small ventro-lateral buds posterior to the prototroch. A further day's growth and they reached the comparative size shown in Figure 4. The eyes were larger and were brown in colour while a pair of large brown pigment patches anterior and lateral to them were a conspicuous feature of the head. These patches varied greatly among different individuals, some were without them altogether, others had a patch on one side only. According to Herpin they are developed out of the pink pigment of the prototrochal region which no longer formed a band below

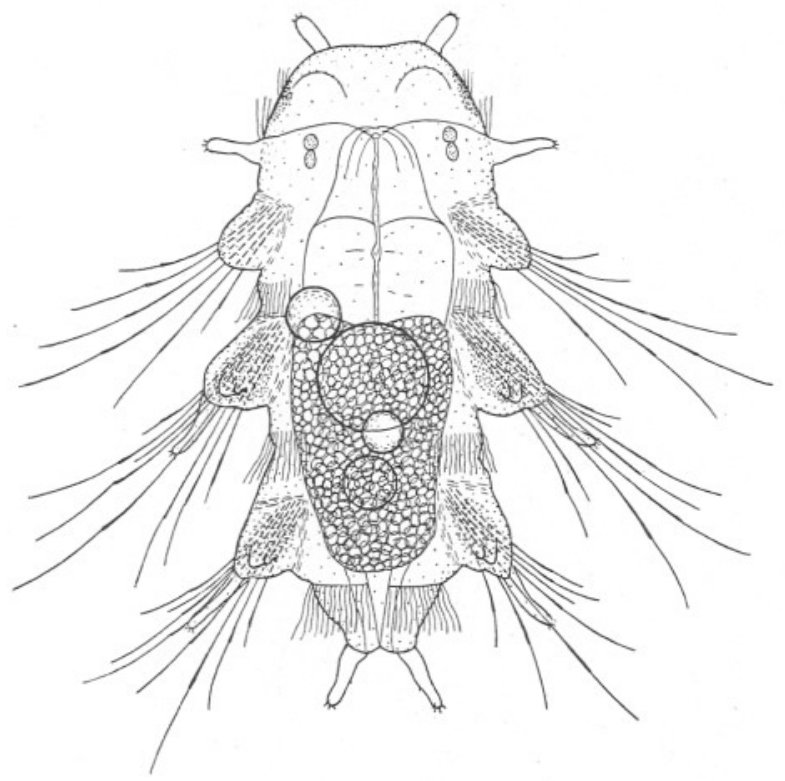

Fig. 5.-Ventral view of larva about 9 days old. $\times 156$. Actual length (exclusive of tentacles and caudal cirri) approx. $380 \mu$.

that organ at this stage. The dorsal gap in the prototroch had widened since the last stage, but the larvæ still swam actively although at times they crawled. For the next few days they could either swim or crawl, but as the cilia gradually disappeared crawling superseded swimming until by the time the stage shown in Figure 6 was reached they were almost entirely crawlers, although they still swam occasionally for short distances along the bottom. When swimming the chætæ were laid along and pressed close against the body with their tips directed backwards.

We must now return to consider the stage shown in ventral view in Figure 5. This larva, about nine days after fertilisation, and two days older than the one whose head is shown in Figure 4, is specially interesting. 
The prototroch had disappeared except for a few cilia on either side of the head, and the akrotroch was likewise confined to two short lateral tracts. The first paratroch showed little or no change, but the second had reacquired the wide ventral gap shown in the figure. The third paratroch had a slight ventral gap but was usually complete dorsally. In the specimen here illustrated the brown pigment on the sides of the head was granular. The eyes could be seen through the transparent head. The tentacles and tentacular cirri were lengthening and the palps had

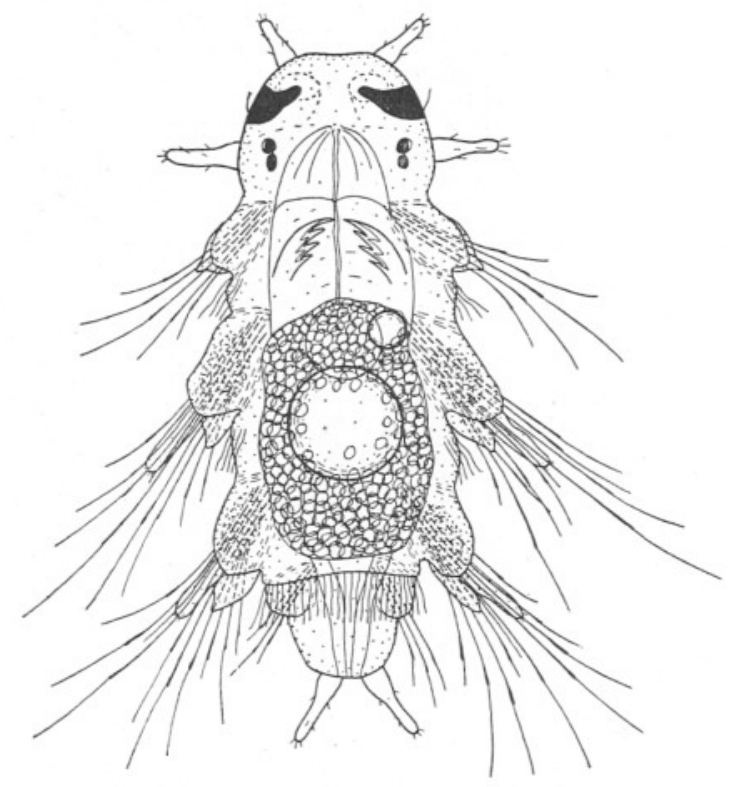

FIG. 6.-Dorsal view of larva 18 days old. $\times 156$. Actual length (exclusive of tentacles and caudal cirri) approx. $400 \mu$.

appeared on the under surface of the head anterior to the line of the prototroch. The first pair of parapodia still consisted of noto- and neuropodial sacs only, but the second and third pairs in addition to the now long middle lobe showed the rudiments of the ventral lobe and of the ventral cirrus (see also Fig. 7). The dorsal cirrus did not appear until considerably later. In the middle of each parapodium of the last two pairs there was a mass of granular tissue constituting, apparently, a gland. This mass became very conspicuous and the granules highly refringent when the living creature was put into a drop of saliva. The anal cirri were fairly long. The buccal and pharyngeal regions were marked out, but the middle portion of the gut was still very granular and with it were 
closely associated the large oil globules which had shifted backwards from the anterior position they occupied during the earlier stages.

A day or two after the last stage the jaws became visible and chætæ of a fourth pair of parapodia appeared posterior to the third paratroch. Growth for the next few days was rather slow and at eighteen days old (Fig. 6) the larva was only a little further advanced than it was at nine days (Fig. 5). The jaws and fourth pair of parapodia were conspicuous, the neuropodium of the second and third pairs of parapodia was larger, and a central lobe was growing out between the bristle bundles of the first

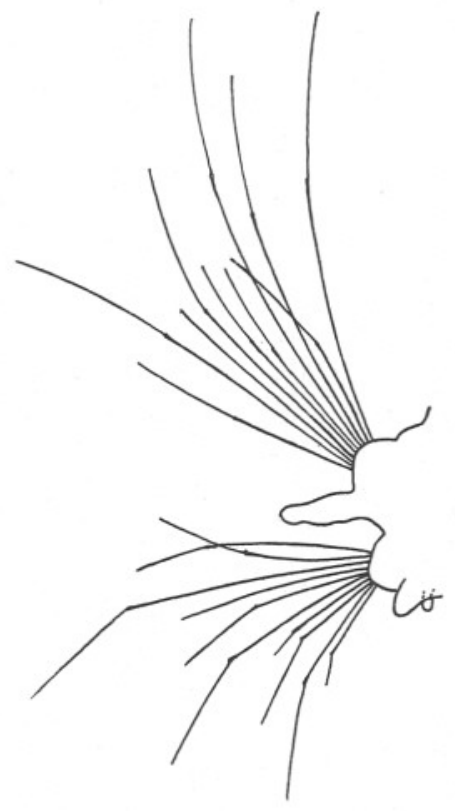

Fis. 7.-Outline of second parapodium of right side, viewed from in front, of a larva about 10 days old. $\times 270$.

pair. The ciliation was still more reduced, the prototroch had quite gone, the akrotroch almost so, while the first and second paratrochs were only short lateral rows. Only the third paratroch had undergone little or no change. Growth continued slowly. The palps became fairly well developed and mobile. The buds of a second pair of tentacular cirri arose ventral to the first pair. The middle lobe of the first pair of parapodia lengthened and the bristles of this pair began to fall out. The bristles with long terminal appendices of the other parapodia were being replaced by bristles with shorter terminal appendices. Dorsal cirri appeared on the second, third, and fourth notopodia, although in some cases on the second they lagged behind those developed on the posterior 
two pairs. Thus was reached the condition shown in Figure 8 of a larva thirty-four days old. It will be noticed that the oil globules were much smaller than formerly and the middle tissues of the gut less granular. The introvert was protrusible and the creature was feeding, the stomach containing a brownish mass of diatomaceous material. The cilia had finally all disappeared. Figure 9 is of a still more advanced stage, but the

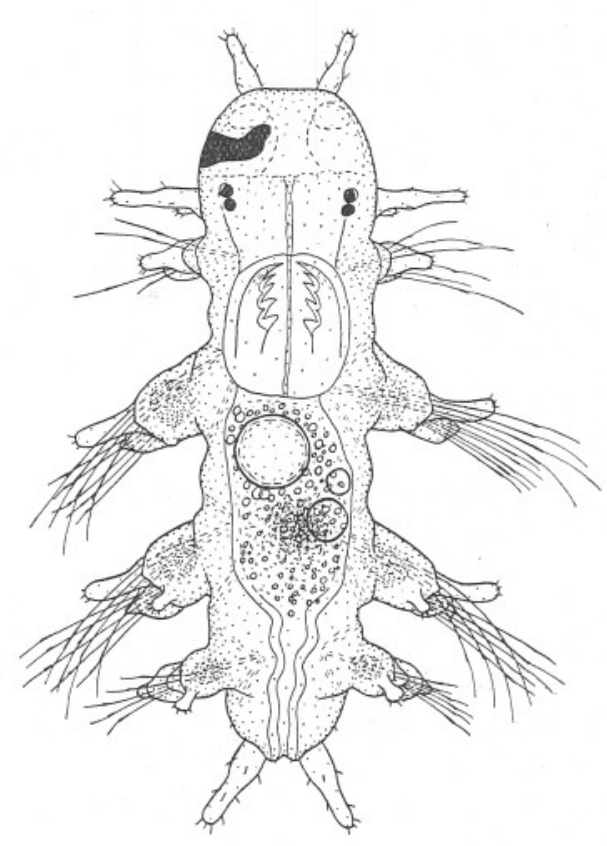

Fig. 8.-Dorsal view of a larva 34 days old. $\times 156$. Actual length (exclusive of tentacles and caudal cirri) approx. $430 \mu$.

FIG. 9.-Dorsal view of a young worm 33 days old. $\times 156$. Actual length (exclusive of tentacles and caudal cirri) approx. $560 \mu$.

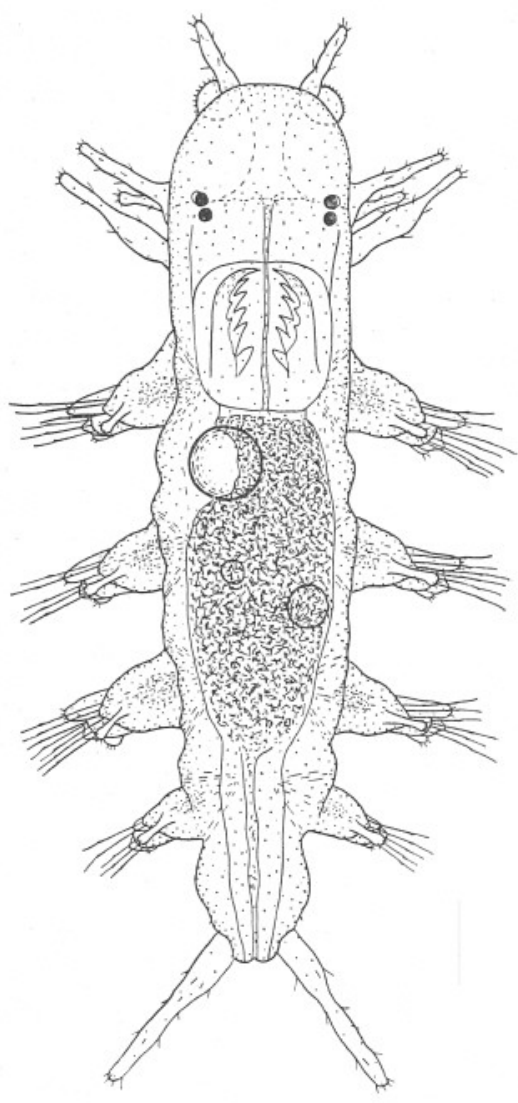

Fr. 9 .

specimen was actually a day younger. Great variation existed as to the state of development of different individuals at this time, some having grown much faster than others. There was also variation in the stages at which different organs appeared; thus in some the first pair of parapodia lost their bristles before the fifth pair of parapodia were visible, in others afterwards. The worm shown in Figure 9 had lost the bristles of the first pair and the middle lobe on each side formed a long tentacular cirrus and was directed forwards just behind the first two pairs. The fifth larval pair 
of parapodia was well developed, making in all four adult pairs. The last of the bristles with long appendices had fallen out. The stomach was full of diatomaceous material, although oil globules were still present.

Individuals continued to grow at very different rates, and some were definitely monstrous with malformed heads, etc. Of those which grew the most rapidly one worm had seven adult pairs of parapodia and the eighth pair forming forty days after fertilization. Seven days later two specimens were seen with eight pairs and the ninth and tenth forming. The brown pigment patches on the head had disappeared and the prostomium was acquiring the adult shape. On the same day as the last was seen a specimen with ten pairs of adult parapodia and the eleventh and twelfth pairs forming. In this specimen the buds of the fourth pair of tentacular cirri could be distinguished ventral to the third pair, the latter being those which had originally developed as lobes between the bristle bundles of the first pair of larval parapodia and which by now were much the longest of the tentacular cirri. Figure 10 shows a young worm sixty-five days old. It had twenty-one pairs of parapodia with the twenty-second, twenty-third, and twenty-fourth in process of formation. It is interesting to note that in these the dorsal cirrus is almost the first part to appear, in the anterior two or three pairs it was almost the last. An outline of the tenth parapodium of a worm at this stage is given in Figure 12. All the adult lobes were present, but it had not quite reached the adult shape. The general appearance of the worm was very like that of the adult except that from above the segments looked squarer. Paragnaths were appearing but were very difficult to see. These young worms were living in tubes which they had formed and fastened to the bottom of the plunger-jar. The walls of the tube were of a parchment con-

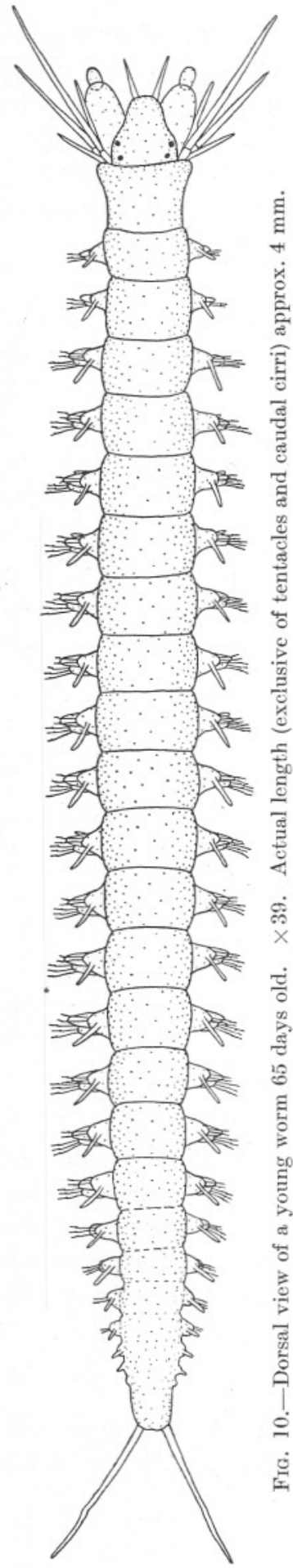


sistency. They were feeding on diatoms. By the end of July, or about five and a half months after fertilisation, some of the worms had reached an average length of one centimeter and had forty to fifty chætigerous segments. The paragnaths showed the typical adult pattern and were easily visible. The following February, one year after fertilisation, the largest of the surviving worms was one and a half

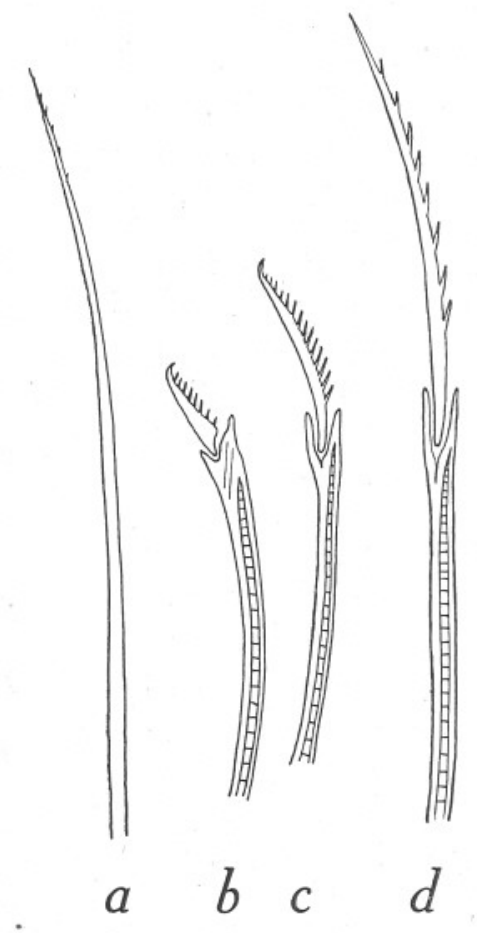

FIG. 11.-Bristles of a larva about 18 days old. $\times 756$. (a) simple capillary, (b) heterogomph falciger, $(c)$ homogomph falciger, $(d)$ homogomph spiniger.

centimeters (measured when fixed) and had approximately sixty chætigerous segments. During the whole year of rearing the water in the plunger-jar was not changed nor was fresh water added to make up for that lost by evaporation. Some of the worms lived for some weeks longer without any great changes and eventually died.

\section{The Succession of the Bristles.}

While reading the following account the Table showing the approximate number of the bristles during the different stages should be consulted. 
In the early stage with three chætigerous segments (Fig. 5) the bristles were mainly homogomph spinigers of the type shown in Figure 11, d. The appendices of these bristles varied in length from approximately $70 \mu$ to $35 \mu$, the long ones predominating, and they were spined. Herpin's figures (1926, Fig. 3, b, c) do not show these spines. The first parapodium had about seven spinigers in its dorsal bundle and about five in its ventral, while the second and third parapodia each carried about ten dorsally and five to seven ventrally. In addition to these all the neuropodia bore about two homogomph falcigers (Fig. 11, c) with spined appendices of approximately $20 \mu$ long. The shorter appendices of the spinigers approached in structure to these falcigers and it is doubtful if there was any great significant difference between them. The dorsal and ventral bundles of the second and third parapodia each contained one simple capillary bristle slightly spined at its distal extremity (Fig. 11, a). The noto- and neuropodia of these segments each had an acicule; no acicules were ever present in the first pair of larval parapodia.

By the time that four chætigerous segments were present (Fig. 6) certain changes had taken place. Homogomph spinigers still predominated, but the dorsal bundle of the first parapodium had now only about four; the second and third, five or six, and of course there was none in the fourth. Moreover, the bristles with the longest appendices had fallen out and the lengths of these now varied from $55 \mu$ to $30 \mu$, and there was a larger proportion of the shorter ones. The dorsal bundle of the fourth parapodium had only one or two (according to stage of development) homogomph falcigers (Fig. 11, c). The ventral bundles of the first three parapodia had lost all their homogomph spinigers except one or two. The first had in addition two homogomph falcigers while the second and third carried three to five of these (Fig. 11, c) as well as two to three heterogomph falcigers (Fig. 11, b) which were not represented in earlier stages. They still retained their single capillary bristle. The ventral bundle of the fourth parapodium had about two homogomph and one heterogomph falcigers.

Table Showing Succession of Bristles (Numbers Approximate).

- Approx. Notopodium stage Neuropodium

Fig. 5.

$\begin{array}{crrrrr}\text { Larval Parapodium Number } & 1 & 2 & 3 & 4 & 5 \\ \text { homogomph spinigers } & 7 & 10 & 10 & & \\ \text { capillary bristle } & - & 1 & 1 & & \\ \text { homogomph spinigers } & 5 & 6 & 6 & & \\ \text { " falcigers } & 2 & 2 & 2 & & \\ \text { capillary bristle } & - & 1 & 1 & & \end{array}$




\begin{tabular}{|c|c|c|c|c|c|c|c|}
\hline \multirow{5}{*}{$\begin{array}{c}\text { Approx. } \\
\text { stage } \\
\text { of }\end{array}$} & \multirow{3}{*}{ Notopodium } & Larval Parapodium Number & 1 & 2 & 3 & 4 & 5 \\
\hline & & homogomph spinigers & 4 & 6 & 6 & - & \\
\hline & & ,, falcigers & - & - & - & 1 & \\
\hline & & capillary bristle & - & 1 & 1 & - & \\
\hline & Neuropodium & homogomph spinigers & 2 & 1 & 2 & - & \\
\hline Fig. 6. & & , falcigers & 2 & 5 & 5 & 2 & \\
\hline & & heterogomph falcigers & - & 2 & 2 & 1 & \\
\hline & & capillary bristle & - & 1 & 1 & - & \\
\hline Approx. & Notopodium & homogomph spinigers & 2 & 3 & 3 & - & \\
\hline stage & & , falcigers & - & - & - & 2 & \\
\hline of & & capillary bristle & - & - & 1 & - & \\
\hline Fig. 8. & Neuropodium & homogomph falcigers & 2 & 5 & 4 & 3 & \\
\hline & & heterogomph falcigers & - & 3 & 3 & 2 & \\
\hline Approx. & Notopodium & homogomph falcigers & - & 2 & 2 & 2 & \\
\hline stage & Neuropodium & homogomph falcigers & - & 4 & 4 & 4 & \\
\hline of & & heterogomph falcigers & - & 3 & 3 & 3 & 2 \\
\hline
\end{tabular}

Fig. 9.

At the transition stage (Fig. 8) the number of homogomph spinigers was still further reduced. The dorsal bundle of the first parapodium (which was rapidly becoming a tentacular cirrus) retained only two or three of the shorter variety, while the ventral bundle had two or three homogomph falcigers. All these were in process of falling out. The second and third parapodia had dorsally three or four homogomph spinigers with appendices of the medium and short lengths, again showing a reduction in number on the previous stage. The capillary bristles were falling out, likewise those of the neuropodium. The dorsal bundle of the fourth parapodium still had two homogomph falcigers. The ventral bundles of the second and third parapodia had lost all their homogomph spinigers but had about five homogomph falcigers and two to four heterogomph falcigers. The ventral bundle of the fourth parapodium had three or four homogomph falcigers and two or three heterogomph falcigers.

Finally, at the stage shown in Figure 9, the first pair of parapodia had lost all their bristles and become tentacular cirri, while the second and third larval parapodia (first and second adult) had shed the last of the homogomph spinigers. Their dorsal bundles, as well as those of the third and fourth adult parapodia, each carried two homogomph falcigers of the type shown in Figure 11, c. The ventral bundles of the first three pairs of parapodia possessed three or four falcigers of the same type and had in addition two or three heterogomph falcigers of the type shown in Figure 
11, b. The neuropodia of the fifth pair each carried one homogomph and two heterogomph falcigers.

I have not followed in detail the subsequent history of the bristles, but a few words on their condition at the stage of Figure 10 will not be out of place. The first two parapodia, which were smaller than the remainder, had lost their dorsal bristles entirely. The remaining parapodia, except those developing at the posterior end, were all very similar to the tenth, which is illustrated in Figure 12. The bristles on the whole corresponded to those found in the adult except that dorsally there were two homogomph falcigers similar to, but of a more robust type than that shown in Figure 11, c. The adult possesses homogomph spinigers in this position and actually some of the more anterior parapodia at this stage had such

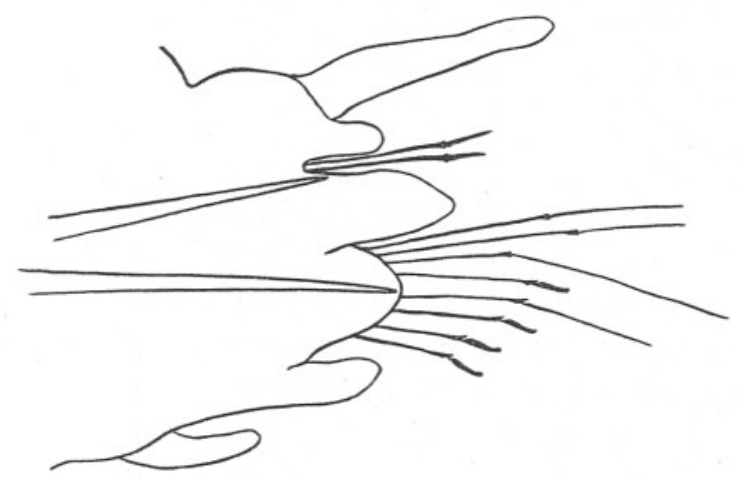

FIG. 12.--Outline of tenth left parapodium of a young worm 66 days old. $\times 270$.

spinigers, suggesting that the falcigers are replaced from in front backwards. The ventral bundle resembled that of the adult; above three homogomph spinigers, below them a heterogomph falciger, then a heterogomph spiniger and finally lowest of all three heterogomph falcigers. All these bristles resembled the adult patterns.

At the age of one year approximately the first twenty parapodia (except the first and second) on either side carried dorsally about three homogomph spinigers of adult type, while the remaining posterior notopodia had only one bristle each, and that a stout homogomph falciger, of the kind found in the same situation in the adult. The ventral bristles were similar to those of full-grown specimens.

\section{Comparison with Herpin's Larve.}

Herpin (1926) has also reared larvæ of $N$. pelagica from the egg, but his specimens behaved somewhat differently from mine. They hatched out at a later stage, one that appears to have been nearly identical with that 
shown in Figure 3. They were then seven days old. While in the egg capsule they had one ciliated girdle, the prototroch, which disappeared before they were actually liberated. Thus on hatching they were incapable of swimming and crawled at once. My larvæ on the other hand were provided with several ciliated girdles, or parts of girdles, and swam strongly for the first few days, only gradually taking to crawling as the cilia were slowly lost. They could indeed still swim a little when eighteen days old (Fig. 6). Herpin's larvæ developed more slowly than mine; the last stage he figures was twenty-eight days old and was, if anything, a little less advanced than my larvæ at eighteen days. He declares that at eighty-three days his larvæ were scarcely more developed than this. In the absence of more abundant data it does not seem justifiable to speculate as to the cause of these interesting differences.

\section{SUMMARY.}

(1) Larvæ of Nereis pelagica Linnæus were reared from the egg, and the young worms, which developed from them, to the age of one year.

(2) The larvæ from an early stage possessed three chætigerous segments. At first after hatching they swam strongly by means of a prototroch, an akrotroch and three paratrochs, but as the cilia gradually disappeared they crawled more and more, finally abandoning swimming altogether.

(3) The head developed a pair of tentacles anteriorly and a pair of palps ventrally. A pair of tentacular cirri arose posterior to the prototroch and a little later a second pair ventral to them.

(4) The first pair of parapodia lost their bristles, and a lobe which had grown out between the noto- and neuropodial chæta-sacs became a third and posterior pair of tentacular cirri. Much later a fourth pair developed ventral to them.

(5) About the time when the larvæ ceased to swim (18 days old) a fourth pair of larval parapodia (third adult pair) appeared, to be followed by a fifth, sixth, etc. At sixty-five days old worms had about twentyone pairs of parapodia, at one year they had sixty and were then about $1.5 \mathrm{~cm}$. long.

(6) During larval development a constant succession of bristles was seen. In the earliest stages the bristles were mainly homogomph spinigers with long appendices; later these fell out and were replaced by homogomph and heterogomph falcigers with shorter appendices. Later still these were replaced by bristles of the adult type.

(7) It is pointed out that these larvæ differed from those which Herpin reared in that they hatched earlier and instead of crawling as soon as liberated they swam for several days by means of their ciliated girdles. 


\section{REFERENCES.}

Herpin, R. (1926.) Recherches biologiques sur la reproduction et le développement de quelques Annélides Polychètes. Bull. Soc. Sci. Nat. de l'Ouest de la France. 4e Série, t. V. (1925).

Wilson, E. B. (1892.) The Cell-lineage of Nereis. Jour. Morph., Vol. VI. 
